\title{
Adaptation de modèles épidémiologiques pour l'analyse des propagations de défaillances dans une ressource de production
}

\section{Adaptation of Epidemiological Models to Study Failures Propagation in a Production Resource}

\author{
F. Ongowe ${ }^{1,2}$, S. Hennequin ${ }^{1}$, A. Nyoungue ${ }^{1, *}$, J. Kagunda ${ }^{2}$, W. Ogana ${ }^{2}$ \\ ${ }^{1}$ LGIPM, Université de Lorraine, Metz, France \{fredrick.ongowe; sophie.hennequin; aime.nyoungue@univ-lorraine.fr\} \\ ${ }^{2}$ School of Mathematics, University of Nairobi, Nairobi, Kenya \{wogana; jwndirangu@uonbi.ac.ke\}
}

*Auteur référent

RÉSUMÉ. Nous explorons ici l'applicabilité de modèles épidémiologiques pour décrire la propagation de défaillances dans une ressource de production. En effet, la complexité et d'interdépendance des systèmes fait qu'une perturbation ne dégrade pas seulement un élément mais peut s'étendre à d'autres éléments, entrainant l'arrêt des systèmes. De plus, une ressource dégradée risque consommer et de générer des pollutions ou des déchets supplémentaires. Notre idée est d'étendre l'application des modèles épidémiologiques aux ressources de production. Dans le secteur de la santé, l'étude de la propagation des pathogènes d'un individu à l'autre a largement contribué au développement des modéles épidémiologiques. Par exemple, le virus $\mathrm{VIH}$ perturbe le fonctionnement normal du corps, passant d'un état robuste à un état léthargique. Dans un contexte industriel, la dégradation des composants des machines perturbe le processus normal de la production avec des états transitoires (qualité moindre, surconsommation d'énergie...) Cette adaptation d'un modèle épidémiologique nous semble prometteuse d'une part pour cibler au mieux la stratégie de maintenance à mettre en œuvre et d'autre part pour évaluer les dynamiques mises en œuvre et représenter au mieux les phénoménes de dégradation intermédiaires (surconsommation d'énergie, dégradation du niveau de qualité, émission de polluants, etc.)

ABSTRACT. We explore the applicability of an epidemiological model to describe failure propagation in an industrial production resource. The complex interdependency in a production system, generally does not only degrade the components, but resultant failure could spread to other performing components, leading to an ultimate collapse of the whole production system. In addition, a degraded production component is characterized by : inefficiency, low output, high energy consumption and production of environmentally unfriendly wastes among others. For instance, analysis of the dynamics of human immunodeficiency virus (HIV) with reference to its propagation resonates well with the failure propagation in a production resource like a production system. The HIV virus causes disruption and degradation to the normal function, the human body system is turned from a robust to a lethargic state. In a similar manner the degradation of a production system's components cause perturbation to the normal production process.

MOTS-CLÉS. Modèle épidémiologique, modèle de type $\mathrm{VIH}$, phénomènes de défaillance, modèle de propagation des défaillances, ressource de production.

KEYWORDS. Epidemiological model, HIV model, faulty phenomena, failures propagation model, production resource.

\section{Introduction}

Dans une installation industrielle, les systèmes et machines se détériorent avec l'âge et l'utilisation. Certains systèmes comme les systèmes militaires, les centrales nucléaires, les unités de soins intensifs, etc. sont beaucoup plus critiques que d'autres. Leur criticité peut conduire à des événements désastreux, y compris la perte de vies humaines en cas de pannes en cours de fonctionnement. C'est pourquoi, de nombreuses recherches ont été menées, notamment sur la base de simulations, pour analyser les performances des systèmes de production soumis à des aléas perturbateurs (pannes, surcharges, erreurs, etc.) [Mourani et al. 2007; Smith, 2003]. Cependant, les simulations peuvent prendre beaucoup de temps et les résultats obtenus peuvent être difficiles à interpréter [Banks et al. 2010]. Des études analytiques 
ont également été proposées. Cependant, celles-ci posent de nombreuses hypothèses restrictives ou simplificatrices (même si les méthodes de décomposition et d'agrégation sont utilisées) et se concentrent davantage sur les performances en régime permanent et des optimisations à long terme [Zhao et al. 2015].

La similitude entre les systèmes biologiques et les systèmes industriels a depuis longtemps été reconnue et étudiée. Ce parallèle a permis de mettre en avant que nous devrions aspirer à imiter les écosystèmes naturels, qui fonctionnent de manière efficace, recyclant les matériaux et émettant peu de déchets [Andrews, 1999]. Concernant notre problématique, la propagation d'un virus dans un individu vivant nous semble similaire à la propagation de certaines défaillances dans un système inanimé. Ainsi, le virus de l'immunodéficience humaine (VIH) peut entraîner la destruction du système immunitaire humain [Barre-Sinoussi et al. 2004]. Cependant, l'immunité du corps humain contre le VIH est stimulée par l'administration de la thérapie antirétrovirale [Marin et al. 2009]. Si le corps humain infecté est laissé sans surveillance, le virus VIH attaquera toutes les cellules responsables de l'immunité. Le résultat final sera un corps très malade qui ne peut plus répondre pleinement à ses fonctions. Le corps sera alors sensible à tout agent pathogène externe même si celui-ci est faible et sans action sur un corps sain. Cette dynamique peut être rapprochée de celle de la propagation des défaillances dans un système industriel. C'est pourquoi, l'objectif principal de cet article est d'adapter un modèle de propagation du VIH pour modéliser la propagation de défaillances dans une ressource de production qui pourrait être par exemple un usinage spécifique ou un dispositif plus complexe associant divers éléments. En effet, nous voulons définir un modèle de propagation permettant de décrire comment des perturbations (qu'elles soient externes ou internes à la ressource) peuvent affecter le système en totalité ou en partie et ainsi réussir à anticiper les différents risques.

Le reste de l'article est organisé comme suit. Dans la section 2, nous rappelons la littérature sur les modèles de propagation de perturbations dans des systèmes industriels complexes. Puis, nous présentons un parallèle avec les modèles épidémiologiques et fournissons quelques exemples d'application pour des systèmes industriels. Ensuite, dans la section 3, nous exposons une analogie entre la propagation du virus VIH dans le système du corps humain et une propagation de perturbations / défaillances dans une ressource de production. Sur la base de cette analogie, dans la section 4, nous proposons un modèle de propagation de défaillances pour une ressource de production composée de différents types de composants. Le modèle proposé est couplé à une analyse du phénomène de la propagation. Dans la section 5, une analyse numérique est proposée. Les courbes obtenues sont données par catégorie de composants et en fonction de la propagation afin d'expliquer les phénomènes entourant le fonctionnement du modèle. Les résultats obtenus dépendent bien évidemment des paramètres choisis pour le modèle, mais ils permettent tout de même de mettre en évidence l'intérêt de celui-ci. Nous concluons cet article et donnons quelques perspectives à notre travail et aux domaines d'application possibles.

\section{Etat de l'art}

Dans cette partie, nous étudions tout d'abord les principaux modèles de propagation de phénomènes perturbateurs pour des systèmes industriels. Puis, nous présentons le principe de fonctionnement des modèles épidémiologiques ainsi que leur adaptation à des systèmes non vivants. 


\subsection{Systèmes industriels complexes}

Le fonctionnement d'un système de production, consiste en une succession d'états allant d'un fonctionnement normal avec un rendement élevé à des états de dysfonctionnement plus ou moins importants (défauts de qualité, surconsommation d'énergie. . . ) jusqu'à un arrêt complet. À mesure que les systèmes s'améliorent en technologie et en efficacité, leur complexité et leur sensibilité s'accroissent et donc leur criticité augmente. De plus, le couplage de ressources différentes rend les réseaux interdépendants très sensibles aux diverses perturbations qui peuvent survenir, c'est-à-dire que ce couplage augmente leur vulnérabilité aux aléas [Buldyrev et al. 2010; Havlin et al. 2010]. Par exemple, la panne d'électricité qui a frappé l'Italie le 28 septembre 2003 était due à deux réseaux : le réseau électrique (utilisé pour le contrôle de surveillance) et le réseau Internet (utilisé pour le système d'acquisition des données [Sturaro et al. 2016; Rosato et al. 2008]. La nature des systèmes et leur mode de fonctionnement génèrent donc des dégradations de certains composants plus sensibles, pouvant créer un défaut de fonctionnement. Le défaut se propage alors aux composants périphériques puis à tout le système. Il est donc essentiel d'identifier ces modes de défaillances et leur propagation. Plusieurs auteurs se sont intéressés à la caractérisation du type de défaillances à l'aide de modèles fiabilistes (bayésiens, markoviens...) [Zhao et al. 2016].

Dans ce travail, nous nous interessons plutôt aux dynamiques de propagation des défaillances. Pendant la période transitoire de dégradation, le rendement des machines se détériore. La transmission des défaillances à d'autres composants entraîne une panne qui arrête finalement la machine et interrompt tout le processus de production. L'étude de ces phénomènes et de leur dynamique de propagation dans le système allant jusqu'à l'arrêt est cruciale car elle joue un rôle important dans la réduction des arrêts de production et la minimisation des coûts. Une réduction des défaillances d'une ressource de production permet non seulement d'éviter les pannes de composants, mais garantit également la fiabilité, la disponibilité et la production optimale.

Les dégradations dépendantes de l'utilisation de la ressource ou issues de facteurs externes et des interactions entre les composants entraîne des taux de production plus faibles générant des coûts de production par article plus élevés avec des niveaux de qualité des produits moindre que ceux prévus sans dégradation de la ressource [Iravani et al. 2002]. De même, pour les unités d'exploitation continues (comme les centrales électriques), la perte de la production est souvent très importante en cas d'arrêt inattendu, beaucoup plus importante que le seul coût d'arrêt de production car le redémarrage par exemple peut être compliqué et coûteux.

Depuis les travaux fondateurs de [Buzacott et al. 1978] sur la performance et l'évaluation des chaînes de production soumises à des défaillances aléatoires, plusieurs modèles ont été proposés. Ces modèles sont soit analytiques, sous la forme de systèmes d'équations généralement simplifiés et pour des configurations très limitées afin de pouvoir conduire l'analyse mathématique complète; soit basés sur la simulation (modélisation type événements discrets à base d'automates d'état du système d'évolution ou de réseaux de Pétri, de chaînes de Markov). Par ailleurs, les principales méthodes d'analyse de la propagation des défaillances disponibles dans la littérature sont menées sous diverses approches. Ainsi, des méthodes quantitatives telles que l'analyse des modes de défaillances, de leur effet et criticité (AMDEC) ou l'analyse des arbres de défaillances sont définies. Ou, des graphes orientés de propagation des défauts, tels que les graphes de propagation de défaillances temporisés (TFPG) sont utilisés. Ces approches sont généralement basées sur l'identification qualitative des défaillances, des causes et des conséquences 
qui aboutissent finalement à leur évaluation quantitative. En plus de représenter la dynamique de la propagation des défaillances dans un système, les graphes orientés décrivent également l'occurrence des pannes, leurs effets locaux et les conséquences sur d'autres parties du système. Dans l'étude de TFPG par exemple, d'après [Abdelwahed et al. 2009], les nœuds et leurs liens représentent les modes de défaillance et la dépendance temporelle entre les nœuds. Les liens sont définis avec des limites inférieures et supérieures du délai de propagation et avec des étiquettes indiquant les modes du système où la propagation correspondante est possible. Ces modèles sont largement développés dans l'analyse de la propagation des défaillances [Zhao et al. 2016]. La troisième famille d'approches décrit les systèmes de production en classant leurs composants sous la forme de structures en série, en parallèle ou selon une combinaison des deux. Cette famille d'approches est plus sure mais plus complexe à mettre en œuvre en termes de simulation et d'analyse.

Cependant, les approches précédentes utilisées pour analyser la propagation de défaillances ne fournissent pas une analyse qualitative et progressive claire de la défaillance par rapport au temps dans le système. Autrement dit, dans ces approches, l'analyse dynamique n'est pas réellement traitée. Il pourrait être intéressant, non seulement de diagnostiquer la propagation des défaillances dans le système de production, mais aussi de suivre l'évolution de cette propagation. Notre idée est d'utiliser un modèle dynamique décrit par un ensemble d'équations différentielles ordinaires (EDO). Dans une ressource de production, les variables considérées représentent les composants de cette ressource évoluant dans le temps. En raison de la propagation d'un défaut, tous les composants n'auront pas la même efficacité. Les composants présentant des caractéristiques similaires en termes d'efficacité et de fiabilité seront classés dans une même classe. Les modèles dynamiques ont connu un attrait important en épidémiologie. L'étude des propagations de maladies, des soins à apporter et de toutes les questions liées à la santé a été largement réalisée à l'aide de ces modèles dynamiques. Nous souhaitons donc utiliser ce type de modèles pour modéliser la propagation dynamique des défaillances. Dans ce qui suit, nous présentons le principe de fonctionnement de ces modèles.

\subsection{Dynamique de propagation des épidémies}

Une maladie infectieuse a la capacité de se propager dans une population à des niveaux intolérables si elle n'est pas contrôlée par un éventuel traitement ou vaccin. L'infection se propage par des interactions entre les agents infectés et leurs voisins sensibles. L'état de la maladie de chaque agent à l'instant $t$ est représenté par une variable. Chaque variable forme une classe qui permet d'indiquer les différents états des individus vis-à-vis de la propagation de la maladie au sein de la population cible. Par exemple, si la population cible subit deux stades différents de propagation, c'est-à-dire $S \rightarrow I$, alors on parle de modèle SI. Ce modèle illustre une méthode commune de modélisation de l'interaction de deux populations, à savoir la classe $S$ (pour susceptible ou sensible), qui représente les individus vulnérables à l'infection. Dans ce compartiment, l'individu / hôte est seulement susceptible d'être infecté car aucun pathogène n'est réellement présent. La classe $I$ représente celle des individus infectés par la maladie et infectieux. Le développement de la maladie augmente avec le temps dans l'hôte. Les modèles SI sont utiles pour certaines infections de plantes car ils représentent le fait qu'un hôte reste infecté jusqu'à sa mort [Haran, 2009].

En dehors du modèle $S I$, il existe d'autres modèles de propagation de maladies comportant plus de deux classes. Ainsi, une population qui subit au moins trois étapes pour compléter le cycle de la maladie peut être représentée par un modèle $S I R$, un modèle SIS ou un modèle SEIR. Dans le modèle SIR, la maladie 
deviendra une épidémie si le taux d'infection est suffisamment supérieur au taux de guérison [Newman, 2010]. Le compartiment $R$ comprend les individus qui étaient autrefois infectés mais qui ont été enlevés soit par une immunité permanente, soit par la mort. Ces individus ne peuvent ni être réinfectées ni transmettre l'infection [Brauer, 2008; Just et al. 2015]. Le modèle SIS peut être dérivé du modèle SIR lorsque les individus infectés se rétablissent sans adopter d'immunité à la maladie. Cela est possible en raison de l'affaiblissement de l'immunité avec le temps, la période d'exposition accrue, l'accoutumance aux médicaments, etc. Le modèle SEIR diffère sur un aspect, c'est-à-dire qu'il catégorise l'hôte sur sa capacité à transmettre l'agent pathogène à un individu sensible. Pour les personnes hôtes qui se trouvent dans la classe $E$ (exposé), leur niveau de santé physique est sans importance. En effet, une personne qui se sent parfaitement en bonne santé peut excréter d'importants éléments pathogènes. Il est donc important de tenir compte du fait qu'en réalité, les frontières entre exposés et infectieux, et, infectieux et récupérés sont floues parce que la capacité de transmettre une infection n'est pas binaire [Haran, 2009]. Il est évident qu'un modèle spécifique ne peut pas être utilisé pour analyser toutes les maladies, car tous les pathogènes n'ont pas la même dynamique de propagation. En plus de dépeindre une propagation différente, des maladies différentes nécessitent des spécifications particulières.

Avec l'application du modèle épidémiologique pour analyser la propagation par étapes des agents pathogènes, des mesures d'atténuation précoces sont également explorées. De plus, les modèles épidémiologiques ont été étendus pour étudier la propagation des menaces dans des objets non vivants. Ainsi, les modèles épidémiologiques ont franchi les frontières de la santé et de la biologie, mais toujours selon le même principe de fonctionnement. Dans ce qui suit, nous présentons quelques exemples d'application des modèles épidémiologiques à des domaines différents de la santé.

\subsection{Applications de modèles épidémiologiques aux systèmes non biologiques}

La perspective de propagation des agents pathogènes dans les organismes vivants est similaire au comportement de menaces pour des éléments inanimés. Le principal phénomène sous-jacent est la nature de l'évolution de la menace. C'est pourquoi, l'application de modèles épidémiologiques pour l'étude de propagation de menaces a été traitée pour différentes applications : informatique, réseaux (informatique ou électriques), économie et finance, pour n'en citer que quelque unes. Les premières applications des modèles épidémiologiques ont été faites dans le secteur de l'informatique avec l'analyse de la propagation de virus informatiques et l'étude de réseaux peer-to-peer. Le virus informatique est la principale menace sur Internet, et son apparition et sa propagation peuvent avoir un impact extrêmement négatif pour les utilisateurs. Le désir de comprendre le mécanisme de propagation de ces virus a inspiré la proposition d'une variété de modèles épidémiologiques basés généralement sur des réseaux entièrement connectés [Rodrigues, 2016]. En complément, la recherche [Piqueira et al. 2005], montre la dynamique de la propagation de l'infection virale provoquant une défaillance dans un réseau d'ordinateurs. Le modèle contient une modification liée au modèle SIR traditionnel, par une classe d'éléments avec antidote représentant des nœuds du réseau informatique équipés de programmes antivirus efficaces. Le modèle est connu sous le nom de SAIR dont les dynamiques sont étudiées de manière similaire à celle des système biologiques.

L'application du modèle épidémiologique dans l'étude de réseaux tels que : les réseaux sociaux en ligne, le déploiement du marketing en utilisant les nouveaux outils informatiques (aussi connu comme marketing «viral »), les réactions du public et la diffusion des idées sur Internet sont également un thème développé. Ainsi, plusieurs articles ont abordé à l'aide des modèles épidémiologiques la possi- 
bilité d'adoption ou d'abandon de l'utilisation des réseaux sociaux en ligne tels que Facebook, Twitter, Instagram parmi d'autres dans les stratégies des entreprises (et des Etats). Cannarella et Spechler ont étudié la diffusion de l'information sur Twitter afin de comprendre les propriétés des médias sous-jacents et les modèles de communication [Cannarella et al. 2014]. Le modéle SIR a également été utilisé dans l'étude de la propagation des rumeurs à l'aide de la technologie de microblogues (vidéos très courtes diffusées sur le net) [Wang et al. 2014]. Le marketing viral est largement utilisé pour populariser et vendre des produits sur les plates-formes en ligne dans le but de persuader les clients d'acheter des produits spécifiques. Lorsqu'une publicité marketing devient virale, elle est analogue à une épidémie, car elle implique la transmission d'un message de la part d'une personne qui se répand finalement dans une population [Kandhway et al. 2014; Rodrigues et al. 2015].

Le troisième grand secteur d'application se situe dans les domaines de l'économie et de la finance. En effet, les prévisions rationnelles en économie et la propagation d'effets (comme une fusion entre grandes entreprises, une crise économique, etc.) sur les réseaux financiers sont deux secteurs majeurs de l'économie. Les prévisions rationnelles explorent la fusion entre l'épidémiologie économique et les modèles épidémiologiques traduisent alors une prise de décision rationnelle. Cette prise de décision rationnelle ainsi mise en évidence permet d'importants progrès comme par exemple la formation des responsable de santé vis-à-vis des implications comportementales lors de la mise en œuvre de politiques publiques de santé (campagne de vaccinations... ). De même, les études de propagation dans les réseaux financiers permettent de mettre en évidence la «contamination » du réseau interbancaire qui peut donc ainsi être considérée comme un modèle épidémiologique [Rodrigues, 2016].

Les modèles épidémiologiques sont largement utilisés dans l'étude de la propagation de maladies, mais également pour l'étude de la propagation de phénomènes liés à différents effets dans des organismes non vivants comme la propagation de logiciels malveillants dans les systèmes informatiques et dans des systèmes interdépendants complexes tels que les réseaux électriques et les réseaux de communication. Notre travail s'inscript dans ce cadre et tente d'adapter le principe du modèle épidémiologique pour décrire la propagation d'une défaillance dans une ressource de production. A partir du pool de modèles épidémiologiques, nous nous sommes intéressés plus particulièrement à la recherche effectuée par [Arruda et al. 2015], dont la recherche exposait un modèle épidémiologique décrivant la propagation du VIH dans le corps humain avec une approche de contrôle optimal de l'immunoloqie du VIH. Avant de proposer notre adaptation, il nous semble judicieux de commencer par présenter une analogie entre un système vivant comme le corps humain et une ressource de production.

\section{Analogie}

La propagation et les effets du virus VIH dans une population sont similaires aux effets résultant de la propagation d'une défaillance dans une ressource de production. Une population à forte prévalence du VIH est affaiblie et improductive d'autant plus si aucune aide extérieure / médicamentation n'est mise en œuvre car une partie des cellules au moins est anéantie par la pandémie. D'un autre côté, une ressource de production robuste et fiable peut subir une dégradation entraînant une défaillance si la maintenance n'est pas effectuée efficacement et rapidement. Une machine ou un composant défaillant (dégradé) est un élément obsolète qui ne fonctionne pas à la capacité optimale prévue par le fabricant. Cette analogie nous a amené à trouver une corrélation entre la propagation de la maladie et la propagation de défaillances 
dans une ressource de production industrielle. Cette section se décompose comme suit : l'applicabilité d'un modèle épidémiologique de type VIH est présentée, puis les similarités et enfin les disparités avec une ressource de production.

\section{Applicabilité du modèle épidémiologique $\mathrm{VIH}$ aux ressources de production :}

Le modèle épidémiologique de propagation du VIH permet d'évaluer la propagation du virus dans les cellules du corps si cette propagation n'est pas limitée voire supprimée par l'utilisation de trithérapie. De même, les défaillances dans les ressources de production peuvent entraîner des effets dévastateurs si aucune maintenance n'est effectuée. Le but du modèle VIH est d'indiquer de quelle manière l'individu infecté peut reprendre ses activités et vivre de manière optimale. La ressource de production, doit être maintenue de manière à fonctionner de manière optimale à la période requise, comme prévu et si possible sans tomber en panne.

Le modèle VIH, peut être étudié par simulation et des conclusions sur la propagation de la maladie à l'ensemble de la population de cellules peuvent être identifiées par l'indication d'un indice de reproduction, $R_{0}$ comme indice seuil. Si cela est adopté pour un modèle de production industrielle, la défaillance entraînant une réduction de la production peut également être analysée de la même manière avec un niveau seuil au-dessus duquel la ressource ne sera plus en état de produire en en deçà duquel elle peut continuer à produire mais avec des résultats non optimaux. Ainsi, les organismes biologiques et les systèmes de production partagent de nombreuses caractéristiques qui sont décrites dans ce qui suit.

\section{Similarités :}

Attaquer les défenses - le taux de mortalité à 100\% réside dans le comportement d'un virus à l'intérieur du corps humain. En effet, à partir de ce comportement, le corps pourrait être désarmé, affaibli et devenir moins bien équipé pour se protéger contre toute forme de maladie. Si l'on compare ces phénomènes avec la propagation d'une défaillance dans une ressource de production, l'adoption de la propagation du VIH se révèle très intéressante.

Répliquer - dans le corps humain, lorsqu'une seule cellule (sensible) est attaquée par exemple par le VIH, le virus se réplique et infecte d'autres cellules. Ceci peut être une similitude de ce qui se passe dans une ressource de production complexe, quand un composant tombe en panne, le reste du système s'arrêtera à cause de la surcharge des parties restantes.

Laisser des vecteurs secondaires faire le travail - pour les individus qui meurent du sida, le VIH est l'infection initiale, c'est-à-dire qu'une fois que le VIH désarme le corps de sa capacité à se défendre, il rend le corps vulnérable aux autres infections dont les symptômes n'auraient même pas pu apparaître si les cellules de défense étaient pleinement actives. Un incident analogue se manifeste également dans une ressource de production. La survenance d'une défaillance dans une ressource rompt (désarme) la continuité de l'interdépendance et des liens entre composants. Le niveau élevé de vulnérabilité auquel la ressource de production est exposée entraîne des niveaux de fonctionnement non optimaux; une productivité minimale qui ne peut pas satisfaire pleinement la demande du consommateur, un niveau de qualité médiocre des produits, des émissions nocives pour l'environnement et / ou une surconsommation d'énergie. Cette défaillance peut se produire en raison d'un mauvais entretien, d'une mauvaise installation, d'une surcharge par exemple. Des composants de production fonctionnant initialement à leur capacité 
optimale lors d'une nouvelle installation vont se dégrader avec le temps, cette détérioration couplée à un manque d'inspection pour détecter les composants défectueux peut amener la panne de la ressource.

Supprimer la multiplication : l'utilisation de la trithérapie n'éradique pas complètement le VIH, mais supprime la multiplication du virus à des niveaux non acceptables pour le corps humain. De même, la maintenance est effectuée sur un système dont le niveau de production est inférieur à l'objectif initial. Bien qu'une maintenance parfaite puisse rétablir l'équipement pour qu'il soit aussi bon qu'à l'état neuf, il n'améliore pas le système mais aide la machine à fonctionner à un niveau donné tel que prévu par le fabricant [Wang, 2002].

Être en latence - si le corps humain est infecté par le VIH, il passe par une période dite de latence où le virus se réplique et commence à attaquer tout le système. Ceci est une réplique de ce qui se passe dans une ressource de production. La dégradation de la ressource de production ne se fait pas en une seule fois. Au stade initial, il n'est pas facile, même lors de l'inspection, de diagnostiquer un début de défaillance dans les composants de la ressource. La manifestation d'une défaillance a lieu le plus souvent après une période de temps pendant laquelle la ressource de production ne peut pas répondre totalement à toutes les attentes.

\section{Disparités :}

Vivant et non vivant - les virus VIH sont des entités vivantes qui peuvent se reproduire dans le corps humain. Dans les ressources de production, les origines d'une défaillance ne sont évidemment pas vivantes, mais elles sont le résultat d'erreurs humaines, d'un manque de maintenance, du vieillissement, etc.

Hôte - le VIH possède des caractéristiques génétiques (l'ADN et l'ARN) qui sont infectieuses pour les êtres vivants alors que la défaillance d'une machine est généralement un défaut résultant d'une mauvaise maintenance et /ou une mauvaise exploitation de la machine pouvant conduire à un surmenage de ses composants.

Composants - Le VIH contient du matériel génétique alors que les défaillances dans les systèmes de production résultent de la dégradation des composants de la machine.

Ces analogies intéressantes entre le phénomène de propagation des défaillances dans les systèmes de production et la propagation des maladies justifient notre choix d'appliquer un modèle épidémiologique à la propagation des défaillances pour une ressource de production. Dans ce qui suit, nous discutons de l'adaptation du modèle VIH à notre problème.

\section{Adaptation d'un modèle épidémiologique :}

A partir de l'analogie précédente, nous nous proposons de définir un modèle de propagation des défaillances. Ce modèle découle du modèle présenté dans Ongowe [Ongowe et al., 2017], lui même s'inspirant du modèle de propagation du virus VIH pour lequel nous avons divisé le nombre total de composants d'une ressource de production en classes. Chacune des classes comporte des composants identiques en termes d'état par rapport à la défaillance et la propagation de celle-ci.

Le modèle de type SIP que nous proposons comprend : la classe $S$ des composants sensibles ou sus- 
ceptibles de tomber en panne, la classe $I$ des composants défectueux, et la classe $P$ des composants protégés ou ayant subi une activité de maintenance (voir la Figure 1). Le modèle SIP est donné par le système d'équations différentielles [1]. Le modèle définit les états d'un composant de la ressource depuis le moment de son installation jusqu'à celui de sa panne.

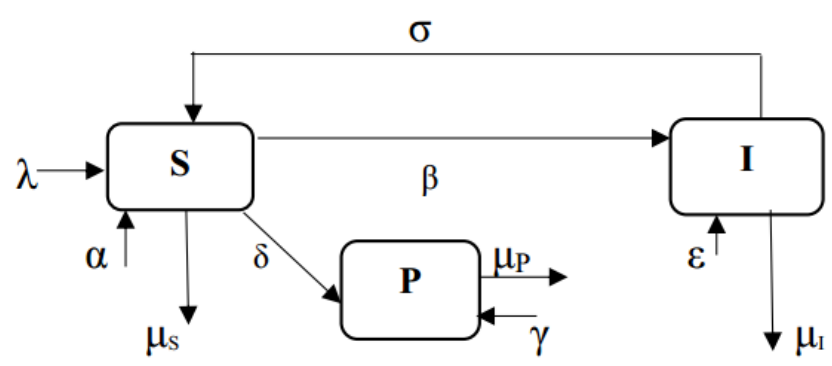

Figure 1.: Modèle de propagation des défaillances

$$
\left.\begin{array}{rl}
\frac{d S}{d t} & =\lambda+\sigma I+\alpha-\left(\beta+\delta+\mu_{S}\right) S \\
\frac{d I}{d t} & =\beta S+\varepsilon-\left(\sigma+\mu_{I}\right) I \\
\frac{d P}{d t} & =\delta S+\gamma-\mu_{P} P
\end{array}\right\}
$$

Pour le modèle SIP proposé, nous ajoutons les hypothèses suivantes :

- un composant protégé, ne peut plus subir de dégradation immédiate menant à une défaillance ;

— si un composant tombe en panne, dans n'importe quel compartiment, il est remplacé immédiatement par un autre avec le même niveau de performances ;

L'évolution dynamique des composants selon la propagation de la défaillance est décrite dans les paragraphes suivants. Ainsi, afin d'essayer d'adapter le modèle épidémiologique décrivant la propagation du VIH au modèle de propagation des défaillances proposé, chaque classe du SIP est identifié en référence au modèle VIH.

\section{Classe des composants susceptibles $S$ :}

La classe $S$ correspond au premier des trois états possibles définis pour les composants d'une ressource de production. Elle renferme l'ensemble des composants qui sont exposés et vulnérables aux phénomènes internes et externes tels que l'usure, la température, la poussière, des vibrations, etc. En raison des facteurs ci-dessus cités, ces composants sont exposés à un taux de dégradation plus élevé pouvant entraîner leur défaillance plus rapidement. De nouveaux composants sont incorporés dans ce compartiment $S$ avec un taux $\lambda$. De plus, via une maintenance parfaite des composants défectueux de la classe $I$, ces derniers peuvent intégrer le compartiment $S$ avec un taux $\sigma$. Selon leur utilisation ou sous l'effet de facteurs externes, les composants du compartiment $S$ peuvent tomber en panne selon un taux $\mu_{S}$. Il seront alors remplacés immédiatement par un composant similaire selon le taux $\alpha$. Les composants sensibles peuvent également, au cours de leur fonctionnement, se dégrader. Ils rejoignent alors le compartiment $I$ (composants défaillants / infectés) avec un taux $\beta$, sinon ils sont protégés et ajoutés dans le compartiment $P$ avec un taux $\delta$. 
Sont définis comme défaillants ou infectés les composants qui du fait de l'utilisation ou selon les perturbations externes, ne fonctionnent pas au niveau souhaité et défini par le fabricant. Ces composants peuvent alors être soumis soit à des activités de maintenance ou à des remplacements en fonction du niveau de la défaillance et de leur nature. Ainsi, les composants de la classe $I$ peuvent subir une maintenance imparfaite via des nettoyages, des réparations, des lubrifications, etc. ou cesser de fonctionner selon un taux de mortalité naturelle $\mu_{I}$. Dans ce dernier cas, ils sont immédiatement remplacés au taux $\varepsilon$, sinon ils seront soumis à la maintenance selon le taux $\sigma$. La nature des actions à mettre en ouvre dépendra donc de l'évolution dynamique des composants dans cette classe.

\section{Classe des composants protégés $P$ :}

Les activités de protection se produisent lorsque le personnel agit sur les composants sensibles en identifiant ceux qui nécessitent une protection supplémentaire. Le choix de la mise en œuvre de cette protection peut se faire par expertise, selon les liens entre composants ou la structure de la ressource. $\mathrm{Si}$ la vulnérabilité du composant à la défaillance est forte, le personnel devra donc installer des dispositifs de protection sur le composant concerné. Cette pratique permet d'intégrer des composants sensibles dans le compartiment $P$ à un taux $\delta$. Au cours de leur fonctionnement, les composants de la classe $P$ peuvent néanmoins également subir des dégradations. Alors, le retrait de ces composants du compartiment $P$ se fait à un taux $\mu_{p}$ et leur remplacement immédiat est effectué au taux $\gamma$.

Si $N$ est le nombre total de composants dans la ressource de production et $M$ la somme des composants dans les trois classes $S, I$ et $P$, à tout instant du fonctionnement de la ressource $M \subseteq N$. Alors la dérivée de $M$ par rapport au temps est donnée par l'équation [2] :

$$
\frac{d M}{d t}=\frac{d S}{d t}+\frac{d I}{d t}+\frac{d P}{d t}=\lambda+\alpha+\varepsilon+\gamma-\left(\mu_{S} S+\mu_{I} I+\mu_{P} P\right)
$$

L'équation [1] indique que le nombre total de composants dans les trois compartiments $M$ n'est pas constant. La combinaison du système [1] avec l'équation [2] conduit au système d'équations [3] suivant.

$$
\left.\begin{array}{rl}
\frac{d S}{d t} & =\lambda+\sigma I+\alpha-\left(\beta+\delta+\mu_{S}\right) S \\
\frac{d I}{d t} & =\beta S+\varepsilon-\left(\sigma+\mu_{I}\right) I \\
\frac{d P}{d t} & =\delta S+\gamma-\mu_{P} P \\
\frac{d M}{d t} & =\lambda+\alpha+\varepsilon+\gamma-\left(\mu_{S} S+\mu_{I} I+\mu_{P} P\right)
\end{array}\right\}
$$

Les variables et paramètres du modèle utilisés pour mettre en évidence la nature propagatrice du système d'équations différentielles [3] sont définis dans le Tableau 4.1 ci-dessous. 


\begin{tabular}{|c|l|}
\hline Variables & Définitions \\
\hline$\lambda$ & Nombre de nouveaux composants recrutés dans $S$ \\
\hline$\delta$ & Taux d'installation de la protection sur les composants $S$ \\
\hline$\sigma$ & Taux de récupération des composants défaillants dans $I$ \\
\hline$\beta$ & Taux de passage des composants $S$ dans la classe $I$ \\
\hline$\mu_{S}$ & Taux de mortalité des composants $S$ \\
\hline$\mu_{I}$ & Taux de mortalité des composants $I$ \\
\hline$\mu_{P}$ & Taux de mortalité des composants $P$ \\
\hline$\alpha$ & Nombre de remplacements des composants défectueux dans $S$ \\
\hline$\gamma$ & Nombre de remplacements des composants défectueux dans $P$ \\
\hline$\varepsilon$ & Nombre de remplacements des composants défectueux dans $I$ \\
\hline
\end{tabular}

Tableau 4.1.: Définition des éléments du modèle

Ainsi, la dynamique du modèle SIP proposé décrit l'évolution des défaillances dans une ressource de production. Dans ce qui suit, nous présentons quelques résultats numériques.

\section{Résultats numériques}

Dans ce paragraphe, une analyse et une simulation numérique sont menées. Le but principal étant d'étudier les changements de comportement du modèle en fonction des tendances prises par les variables. Cette étude est rendue possible par l'utilisation du progiciel Matlab.

\section{1. Étude analytique}

L'étude analytique donnée par le système d'équations [4] considère l'état de stabilité (d'équilibre) comme l'une des principales tendances comportementales du modèle SIP. Cet état est donné par :

$$
\left.\begin{array}{rl}
\lambda+\sigma I+\alpha-\left(\beta+\delta+\mu_{S}\right) S & =0 \\
\beta S+\varepsilon-\left(\sigma+\mu_{I}\right) I & =0 \\
\delta S+\gamma-\mu_{P} P & =0
\end{array}\right\}
$$

À partir du système d'équations [4], la solution des classe $S, I$ et $P$ conduit au système d'équations [5] :

$$
\left.\begin{array}{rl}
S & =\frac{(\lambda+\alpha)\left(\sigma+\mu_{I}\right)+\delta \varepsilon}{\left(\sigma+\mu_{I}\right)\left(\beta+\delta+\mu_{S}\right)-\beta \sigma} \\
I & =\frac{\varepsilon}{\sigma+\mu_{I}}+\frac{\beta}{\sigma+\mu_{I}} \cdot \frac{(\lambda+\alpha)\left(\sigma+\mu_{I}\right)+\delta \varepsilon}{\left(\sigma+\mu_{I}\right)\left(\beta+\delta+\mu_{S}\right)-\beta \sigma} \\
P & =\frac{\gamma}{\mu_{P}}+\frac{\delta}{\mu_{P}} \cdot \frac{(\lambda+\alpha)\left(\sigma+\mu_{I}\right)+\delta \varepsilon}{\left(\sigma+\mu_{I}\right)\left(\beta+\delta+\mu_{S}\right)-\beta \sigma}
\end{array}\right\}
$$


L'ensemble des solutions des variables d'état ainsi obtenues met en évidence l'interdépendance des composants de la ressource.

\subsection{Analyse de la simulation}

L'analyse du modèle SIP est conduite en faisant varier certaines valeurs de paramètres. Les valeurs des paramètres sont choisies ici de façon empirique mais elles pourront facilement être adaptées en fonction de l'analyse de données réelles (historique des pannes, types de composants, expertise. . . ). Les résultats pour différents scénarii sont mis en évidence ci-après.

\begin{tabular}{|c|c|c|c|c|c|}
\hline Paramètres & Estimations & Paramètres & Estimations & Variables & Estimations \\
\hline$\lambda$ & 2 & $\mu_{I}$ & 0.2 & $S$ & 30 \\
\hline$\delta$ & 0,01 & $\mu_{P}$ & 0,4 & $I$ & 20 \\
\hline$\sigma$ & 0,2 & $\alpha$ & 2 & $P$ & 10 \\
\hline$\beta$ & 0,02 & $\gamma$ & 3 & & \\
\hline$\mu_{S}$ & 0,1 & $\varepsilon$ & 2 & & \\
\hline
\end{tabular}

Tableau 5.2.: Valeurs des paramètres et variables

Scénario I : Dans ce premier scénario, les variables et les paramètres utilisés sont ceux du Tableau 5.2 .. Le choix des paramètres a été guidé par l'intention de faire en sorte que le nombre de composants défaillants d'une classe soit supérieur au nombre respectif de composants utilisés pour le remplacement dans la même classe : $\mu_{S} S=0,1 \times 30=3>\alpha=2, \mu_{I} I=0,2 \times 20=4>\varepsilon=2$ et $\mu_{P} P=$ $0,4 \times 10=4>\gamma=3$.

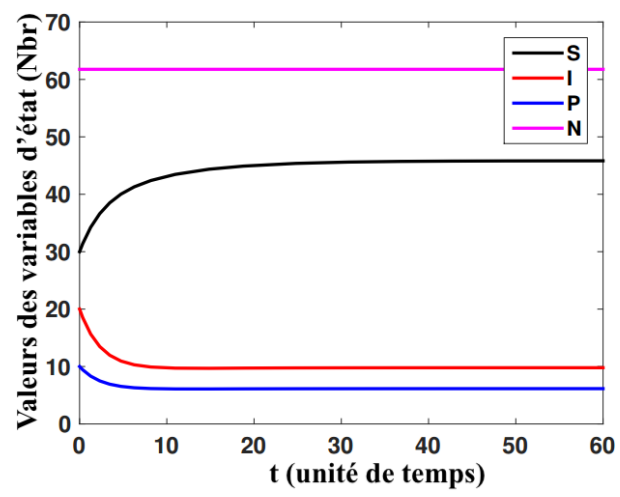

(a) Evolution des variables d'état en fonction du temps (scénario I)

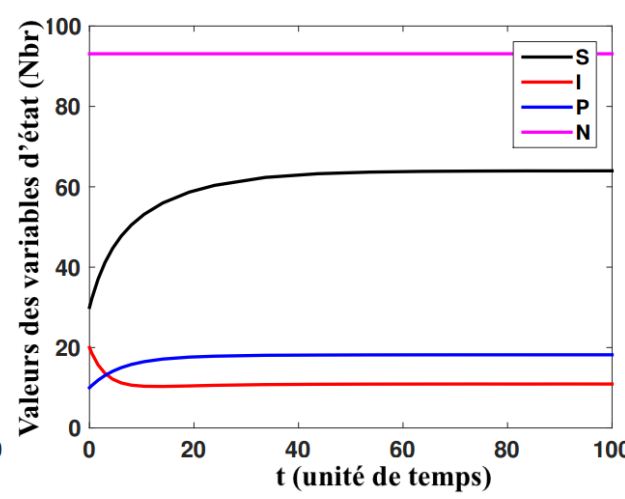

(b) Evolution des variables d'état en fonction du temps (scénario II)

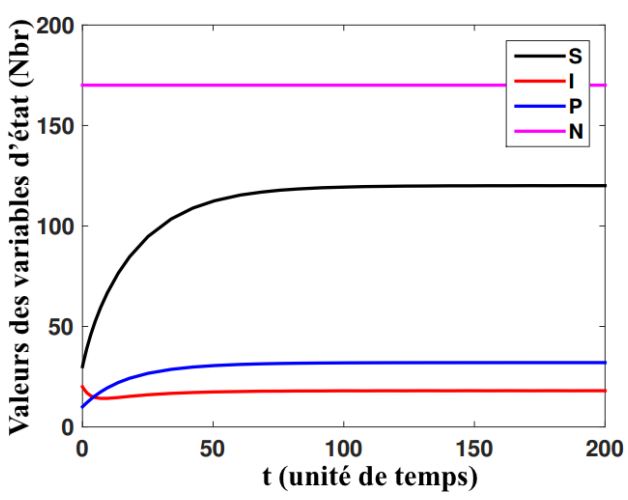

(c) Evolution des variables d'état en fonction du temps (scénario III)

Figure 2.: Evolution des variables d'états en fonction du temps

Au début de la simulation, les composants dans toutes les classes $S, I$ et $P$ augmentent avec le temps avant de se stabiliser comme le montre la Figure 2 a). Bien que $P$ se stabilise à un moment $\mathrm{t} \approx 15$ ( $t$ représentant une unité temporelle pouvant être liée à l'âge de la ressource), $P$ se stabilise à une valeur beaucoup plus élevée que $\mathrm{S}$ et $I$. Le nombre maximum de composants $M(M=S+I+P)$ engagés lors de cette simulation est de 60 unités. 
Scénario II : Les mêmes valeurs de variables et de paramètres sont utiliés mais avec des taux de défaillance différents : $\mu_{S}=0,0667, \mu_{I}=0,1$ et $\mu_{P}=0,2$. L'intention ici est de faire correspondre le nombre de composants défectueux au nombre de composants utilisés dans les remplacements. Les tendances des évolutions générales résultantes des classes $S$ et $I$ sont données dans la Figure 2.b. Dans ce scénario, du fait de l'augmentation des taux de défaillances, il est essentiel de mieux protéger les composants. De plus, la dynamique de propagation est plus lente ce qui laisse supposer de plus grandes interactions entre les classes.

Scénario III : Dans le troisième scénario, nous utilisons les mêmes valeurs de variables et de paramètres, mais nous avons échangé les valeurs des taux de mortalité avec leurs taux de remplacement respectifs. Cela implique que les taux de mortalité sont inférieurs aux taux de remplacement : $\mu_{I} 0,0333, \mu_{I}=0,1$ et $\mu_{p}=0,1$. Le choix arbitraire de tels taux de défaillance pour les différents compartiments permet d'avoir le nombre de composants à remplacer inférieur au nombre de composants remplacés dans chacune des classes. Plus précisément, $\mu_{S} S=0,0333 \times 30 \approx 1<\alpha=2, \mu_{I} I=0,1 \times 20=2<\varepsilon=3$ et $\mu_{P} P=0,1 \times 10=1<\gamma=2$.

L'évolution des variables $S, I$ et $P$ indiquée sur la Figure 2c) est qualitativement similaire aux tendances représentées par les variables de la Figure 2.a) et 2.b) : une tendance croissante suivie d'une stabilité. La valeur totale des composants en jeux dans cette simulation $M \approx 170$ est plus élevée par rapport aux deux premiers scénarios. Dans ce cas, des composants neufs sont introduits en plus grande quantité. Cela génère des interactions fortes entre composant neufs et composants agés entrainant une augmentation importante des composants de la classe $S$.

\section{Conclusion}

Les machines de production ont acquis une sophistication nécessaire pour satisfaire la demande du marché actuel (satisfaction des clients toujours plus forte et concurrence accrue). Ces améliorations résultent en une grande complexité de conception, une haute technicité, une plus grande sensibilité aux impacts environnementaux et sociaux induits et également une plus forte tendance aux défaillances. En effet, la complexité de conception et la nature des opérations à mener rendent souvent la machine plus vulnérable à la propagation de défaillances que les facteurs de perturbation soient internes ou externes. Or, une défaillance est très coûteuse car elle affecte notamment l'efficacité, la production, les coûts de maintenance et de fabrication et la durée de vie de la machine. C'est pourquoi, il est vital de définir des modèles et d'étudier comment la défaillance se propage.

Les principales méthodes utilisées pour étudier la propagation des défaillances, par exemple les arbres de défaillances, sont incapables d'analyser les effets entre le début d'une défaillance, sa propagation, jusqu'au point où la machine tombe en panne. Notre idée est donc d'adapter des modèles d'équations différentielles dynamiques pour représenter la propagation des défaillances comme cela peut se faire pour des systèmes vivants biologiques. En effet, la détection précoce de la défaillance couplée à l'analyse de l'évolution de sa propagation pourrait amener à définir des préventions appropriées. Ainsi, nous avons adapté un modèle épidémiologique basé sur l'analogie présentée entre la propagation du pathogène chez le vivant et la propagation de la défaillance dans une ressource de production. Cette piste de recherche nous semble intéressante pour notre problème car, comme l'indique la littérature, l'application de modèles épidémiologiques n'est pas limitée au seul domaine des êtres vivants. De plus, les compo- 
sants de production peuvent être catégorisés en différentes classes de composants en fonction de leur état de défaillance ou de la façon dont ils sont protégés contre les défaillances comme pour les modèles épidémiologiques.

Partant d'un modèle de propagation du virus VIH adapté à notre problème, nous avons mené une étude de la propagation d'une défaillance dans une ressource en définissant trois classes de composants différentes, à savoir une classe de composants sensibles, une classe de composants défectueux et une classe de composants protégés (pour lesquels les activités de maintenance sont importantes afin de limiter les risques d'arrêt). Dans le modèle proposé, nous avons de plus considéré la mortalité possible de tous les composants et leur remplacement en cas de panne de ceux-ci. Des simulations numériques ont été proposées pour différents cas d'utilisation et donc différents paramètres. Dans ce travail initial, nous avons choisi des paramètres de façon empirique afin de montrer l'intérêt du modèle et ses possibles applications industrielles. Il est à noter qu'au bout d'un certain temps, en raison de la propagation des défaillances, tous les composants atteindront un équilibre, comme le montrent les résultats numériques.

Pour les travaux futurs, nous souhaitons adapter notre modèle avec des données réelles collectées sur une ressource de production (comme une cellule flexible de production). Cela nous permettra de définir d'autres catégories de composants en fonctions des causes de défaillances possibles et des interactions plus ou moins fortes entre composants. Nous souhaitons également affiner notre étude en évaluant les performances de notre modèle selon les valeurs des différents paramètres définis en menant une analyse de sensibilité analytique à l'aide de l'analyse des perturbations bien adaptée à des modèles continus sous forme de système d'équations différentielles. Dans la même idée, nous souhaitons aussi étudier numériquement comment les résultats de notre modèle évoluent lorsque les paramètres deviennent stochastiques ou lorsqu'ils sont définis par des fonctions de différentes variables permettant ainsi de représenter des impacts divers sur les variables définies. Ceci nous permettra également de mieux identifier l'apparition d'une défaillance. Puis, nous souhaitons intégrer dans les états de fonctionnement de la ressource soumise à l'effet de défaillances les notions de surconsommation d'énergie, d'augmentation des polluants et des déchets produits comme cela peut être le cas lorsqu'une ressource n'a pas reçu l'entretien préconisé par le fabricant. Nous proposons également d'appliquer l'approche de la propagation en recommandant les meilleures pratiques de maintenance pour les composants car cela permettrait de réduire les coûts de maintenance, augmenterait la production, et la qualité des produit.

\section{Bibliographie}

Abdelwahed S. Karsai G., Mahadevan N., Ofsthun S. «Practical implementation of diagnosis systems using timed failure propagation graph models. » IEEE Transactions on instrumentation and measurement $58, \mathrm{n}^{\circ} 2,(2009): 240$ $-247$.

ANDREws C. J., «Putting industrial ecology into place evolving roles for planners. » Journal of the American Planning Association. 65, $\mathrm{n}^{\circ} 4$ (1999) : 364-375.

Arruda E. F., Dias C. M., Magalhães C. V., Pastore D. H., Thomé R. C., Yang, H. M., « An optimal control approach to hiv immunology. » Applied Mathematics 6, $\mathrm{n}^{\circ} 06$ (2015) : 1115-1130.

Banks, J., Carson II J., Nelson B., Nicol D., Discrete-event system simulation (5th ed.). Englewood Cliffs, NJ : Prentice Hall. 2010.

Barre-Sinoussi F., Chermann J., Rey F., Nugeyre M., Chamaret S., Gruest J., Dauguet, J., «Isolation of tlymphotropic retrovirus from a patient at risk for acquired immune defficiency syndrome (aids). » Revista de investigación clínica, 56, n 2 (2004) : 126-129. 
BRAUER F., «Compartmental models in epidemiology, 1945, series in lecture notes in mathematics. » In Mathematical epidemiology 65, $\mathrm{n}^{\circ} 4$ (2008) : 19-79.

Buldyrev S. V., Parshani R., Paul G., Stanley, H. E., Havlin S., « Catastrophic cascade of failures in interdependent networks. » Nature 464, $\mathrm{n}^{\circ} 7291$ (2010) : 1025 - 1028.

BUZACOTT J.A., BHANIFIN L. Eo

«Models of automatic transfer lines with inventory banks, a review and comparison. » AIIE Transactions $10 \mathrm{n}^{\circ} 2$ : (1978) : 197 - 207.

CANnARella J., S PeChleR J. A., «Epidemiological modeling of online social network dynamics. » arXiv preprint arXiv :1401.4208. (2014)

HARAN M., «An introduction to models for disease dynamics. » Spatial Epidemiology, SAMSI. (2009)

IRAVANI S.M., DUENYAS I. «Integrated maintenance and production control of a deteriorating production system. »IIE Transactions 34 (2002) : $423-435$.

Just W., CAllender H., «Differential equation models of disease transmission. » (2015) http ://www.ohio.edu/people/just/IONTW/ModuleDE.pdf

KANDHWAY K., KURI J., «Optimal control of sis and sir information epidemics. » Applied Mathematics and Computation. 231, (2014) : 79-92.

Marin B., Thiébaut R., Bucher H. C., Rondeau V., Costagliola D., Dorrucci M., Chene G., « Non-aidsdefining deaths and immunodeficiency in the era of combination antiretroviral therapy. » AIDS. 23, $\mathrm{n}^{\circ}$ 13, (2009) : 1743-1753. DOI : 10.1097/QAD.0b013e32832e9b78

Newman M. E. J., Networks : an introduction. Oxford University Press (2010), Oxford,

Mourani I., HenNeQuin S., XIE X., «Failure models and throughput rate of transfer lines. » International journal of production research. $45, \mathrm{n}^{\circ} 8(2007): 1835-1859$.

Ongowe F., Hennequin S., Kagunda J., Nyoungue A., «Biomathematics modelling for the study of failures propagation : Application to a production resource. » J20th World Congress of International Federation of Automatic Control (IFAC) - July 9-14, 2017 Tolouse, France. (2017)

Piqueira J. R. C., NAvarro B. F., Monteiro L. H. A., «Epidemiological models applied to viruses in computer networks. » Journal of Computer Science. $1, \mathrm{n}^{\circ} 1$ (2005) : 31-34.

RODRIGUeS H. S., FONSECA M. J., «Viral marketing as epidemiological model. » arXiv preprint arXiv :1507.06986. (2015)

RODRIGUES H. S., «Application of sir epidemiological model : new trends. » arXiv preprint arXiv :1611.02565. (2016)

Rosato V., Issacharoff L., Tiriticco F., Meloni S., Porcellinis S., Setola R., «Modelling interdependent infrastructures using interacting dynamical models.. » International Journal of Critical Infrastructures. 4, ${ }^{\circ}$ 1-2 (2008) : $63-79$.

SMith J. S., «Failure Propagation Modeling and Analysis via System Interfaces. » Journal of manufacturing systems. 22, $\mathrm{n}^{\circ} \quad 2(2003): 157-171$.

Sturaro A., Silvestri S., Conti M., DAs S. K., «Towards a realistic model for failure propagation in interdependent networks.. » In Computing, networking and communications (icnc),International Conference (2016)

WANG «A survey of maintenance policies of deteriorating systems. » European journal of operational research. 139, ${ }^{\circ}$ 3 (2002) : 469-489.

WANG J., WANG Y. Q., « Sir rumor spreading model with network medium in complex social networks. » Chinese Journal of Physics.

ZHAO L., LI J., «Analysis and improvement of multiproduct bernoulli serial lines : Theory and application. » IEEE Transactions on Systems, Man, and Cybernetics : Systems, 45, $\mathrm{n}^{\circ} 9$ (2015) : 1218-1230..

Zhao L., Thulasiraman K., Ge X., Nui R., «Failure Propagation Modeling and Analysis via System Interfaces. » Mathematical Problems in Engineering. vol. 2016, Article ID 8593612 (2016). 